\title{
Closed form least-squares solution to 3D symmetric Helmert transformation with rotational invariant covariance structure
}

\author{
Guobin Chang ${ }^{1}$
}

Received: 26 January 2015/ Accepted: 4 June 2015/Published online: 13 June 2015

(C) Akadémiai Kiadó 2015

\begin{abstract}
Least-squares (LS) solutions to the 3D Helmert and symmetric Helmert transformations with rotational invariant covariance structure are studied in a unified framework. This is an extension of the 3D Helmert transformation with naïve identity covariance and the counterpart of the 2D symmetric Helmert transformation with rotational invariant covariance. It is found that the closed form LS solution still exists and that the rotation parameters are still the same between the Helmert and symmetric Helmert transformations.
\end{abstract}

Keywords 3D datum transformation - Symmetric Helmert transformation · Least-squares · Closed form solution · Rotational invariant covariance

\section{Introduction}

In similarity datum transformation, seven parameters, one for scale, three for translation, and three for rotation, are employed to transform the coordinates of one point in one (original) frame into those of the same point in the other (target) frame. The task is estimating the seven parameters using coordinates measured in both frames. In some leastsquares (LS) solutions to this problem, coordinates measured in only one frame are considered subject to measurement errors and thus need to be adjusted, see e.g. (Grafarend and Awange 2003; Sanso 1973; Shen et al. 2006; Yang 1999). This is known as Helmert transformation (Teunissen 1985, 1988). However what is realistic is that coordinates in both frames are subject to errors and hence should be consider as such. This results in the symmetric Helmert transformation (Teunissen 1985, 1988), also known as Gauss-Helmert model (Neitzel 2010).

Guobin Chang

guobinchang@hotmail.com

1 State Key Laboratory of Geo-information Engineering, Xi'an Research Institute of Surveying and Mapping, Xi'an, China 
The 2D symmetric Helmert transformation has already been studied in depth much earlier. In his seminal report (Teunissen 1985), Teunissen pointed out for the first time in the geodetic literature the necessity of considering errors of coordinates in both frames and coined the "symmetric Helmert transformation". The rotational invariant covariance rather than the naïve identity covariance, i.e. isotropic, homogeneous, and uncorrelated, was considered, and closed form LS solutions was derived (Teunissen 1985). The "symmetry" property of the estimators when interchanging the role of the two frames was revealed, and as the covariance for one frame approaches zero, the solution degenerates to that for Helmert transformation (Teunissen 1988). The bias of the solution was also studied in (Teunissen 1989). Besides the similarity transformation, an iterative solution was provided to the $2 \mathrm{D}$ symmetry affine transformation with covariance structure as $\boldsymbol{Q}_{0} \otimes \boldsymbol{I}_{n}$ (Teunissen 1986). A non-iterative solution to $2 \mathrm{D}$ symmetric affine transformation with covariance structure as $\boldsymbol{Q}_{0} \otimes \boldsymbol{Q}$ was derived recently in ( $\mathrm{Li}$ et al. 2013).

The symmetric Helmert transformation, or the Gauss-Helmert model, belongs to the error-in-variable model. The LS solution to this model is often called total least-squares (TLS) solution (Schaffrin and Felus 2008; Schaffrin and Wieser 2008). From (Teunissen 1985), it can be concluded that the TLS is not a new adjustment method but the LS adjustment to a specific nonlinear model. This is widely recognized recently see e.g. (Neitzel 2010; Schaffrin 2006; Xu et al. 2012).

In most of the papers studying the TLS in the geodetic literature, $2 \mathrm{D}$ rather than the more practical 3D transformations are adopted in their case studies. Some of the few exceptions are as follows. Fang (2014) studied the 3D problem with general covariance structure (potentially anisotropic, inhomogeneous, and correlated), however the solution was iterative, and as the rotation parameters are parameterized as Euler angles, there are many transcendental function evaluations involved in each iteration resulting in heavy computational load and potential numerical instability. 3D iterative solutions to 3D symmetric Helmert transformation with inhomogeneous and anisotropic errors are investigated in (Kanatani and Niitsuma 2012a, b). The orthogonal constraint of the rotation matrix is excluded through representing the changes of rotation between successive iterations with 3D infinitesimal rotation (Kanatani and Niitsuma 2012a) while the unit constraints of the quaternion is excluded through consolidating the scale and quaternion (Kanatani and Niitsuma 2012b). In both methods, the transcendental trigonometric function evaluations are avoided in the iterations. Contrast to iterative solutions, Closed form solution to 3D symmetric Helmert transformation has already been derived much earlier in (Teunissen 1985) and an equivalence for the rotation parameters can be found in his solution that the rotation parameters are the same between Helmert and symmetric Helmert transformations. However, in deriving the rotation parameters, the scale parameter was presupposed as fixed. The scale parameter was also presupposed fixed (to be 1) to derive the rotation parameters in (Felus and Burtch 2009), and the above mentioned equivalence was also emphasized there. Recently, Chang (2015) derived the closed form LS solution to the 3D problem without presupposedly fixing the scale parameter, and the equivalence of the rotation parameters was found to still exist in that solution. However, in all the above three, the naïve identity covariance structure was employed.

This paper is concerned with closed form solutions to 3D symmetric Helmert transformation. Of course it is mathematically impossible for arbitrary errors, i.e. inhomogeneous, anisotropic, and correlated ones (Fang 2014), or even inhomogeneous and anisotropic ones (Kanatani and Niitsuma 2012a, b). The rotational invariant covariance structure (Teunissen 1985, 1988) are considered. This structure is more practical than the naïve identity one, and the latter is essentially a special case of the former. The 3D Helmert and symmetric Helmert transformation with this 
covariance structure are studied in a unified framework. It is found that closed form solutions can be found which are optimal in the LS sense. And it is further found that the rotation parameters are the same between the two transformations. These findings, similar to that in (Chang 2015; Felus and Burtch 2009; Teunissen 1985), can be consider as an extension to 3D symmetric Helmert transformation with naïve identity covariance (Chang 2015), and a counterpart of the 2D symmetric Helmert transformation with rotational invariant covariance (Teunissen 1985, 1988).

\section{Main results}

In a similarity model, the coordinates of a point in two Cartesian frames are related as

$$
\boldsymbol{y}_{i}-\boldsymbol{e}_{i}=\boldsymbol{t}+s \boldsymbol{R}\left(\boldsymbol{x}_{i}-\boldsymbol{\varepsilon}_{i}\right)
$$

where $i$ denotes the index of the point, $\boldsymbol{x}_{i}$ and $\boldsymbol{y}_{i}$ are the measured coordinates in the first and second frames, $\boldsymbol{e}_{i}$ and $\boldsymbol{\varepsilon}_{i}$ are the corresponding errors, $s>0, \boldsymbol{t}$, and $\boldsymbol{R}$ are the scale, $3 \times 1$ translation and $3 \times 3$ rotation parameters respectively. Note that $\boldsymbol{R}$ belongs to the 3D special orthogonal group, i.e. $\boldsymbol{R}^{T} \boldsymbol{R}=\boldsymbol{I}$ and $\operatorname{det}(\boldsymbol{R})=1$. The aim is estimating $s, \boldsymbol{t}$, and $\boldsymbol{R}$ given $\boldsymbol{x}_{i}$ and $\boldsymbol{y}_{i}, i=1,2, \ldots, N$. In this study, we assume the measurement errors are isotropic, inhomogeneous, uncorrelated, as follows

$$
\begin{aligned}
E\left[\boldsymbol{e}_{i}\right] & =\mathbf{0}_{3 \times 1} \\
E\left[\boldsymbol{\varepsilon}_{i}\right] & =\mathbf{0}_{3 \times 1} \\
\operatorname{cov}\left[\boldsymbol{e}_{i}, \boldsymbol{e}_{j}\right] & =k_{y} \sigma_{i}^{2} \boldsymbol{I} \delta_{i j} \\
\operatorname{cov}\left[\boldsymbol{\varepsilon}_{i}, \boldsymbol{\varepsilon}_{j}\right] & =k_{x} \sigma_{i}^{2} \boldsymbol{I} \delta_{i j} \\
\operatorname{cov}\left[\boldsymbol{e}_{i}, \boldsymbol{\varepsilon}_{j}\right] & =\mathbf{0}_{3 \times 3}
\end{aligned}
$$

with $k_{x}>0, k_{y}>0$.

In (Teunissen 1985, 1988), the so called rotational invariant structure was employed, it said that

$$
\begin{aligned}
\operatorname{cov}\left[\boldsymbol{e}_{i}, \boldsymbol{e}_{i}\right] & =\boldsymbol{Q}_{i} \\
\operatorname{cov}\left[\boldsymbol{\varepsilon}_{i}, \boldsymbol{\varepsilon}_{i}\right] & =k^{2} \boldsymbol{Q}_{i}
\end{aligned}
$$

and that for any rotation matrix $\mathrm{R}$

$$
\boldsymbol{R} \boldsymbol{Q}_{i} \boldsymbol{R}^{T}=Q_{i}
$$

As $\boldsymbol{Q}_{\mathrm{i}}$ is symmetric and positive-definite, it can only be scaled identity matrix to satisfy (4). So the rotational invariant structure is essentially the same to structure in (2). Note that the naïve identity covariance structure (Chang 2015) is essentially a special case of the rotational invariant structure. Also note that in (2) $k_{x} \rightarrow 0$ implies that only $\boldsymbol{y}_{i}$ are subject to errors and $k_{y} \rightarrow 0$ implies that only $\boldsymbol{x}_{i}$ are subject to errors, so both Helmert and symmetric Helmert model are formulated in a unified framework.

The LS solution is obtained minimizing the following quadratic loss function

$$
L=\sum_{i=1}^{N}\left[\frac{1}{k_{y} \sigma_{i}^{2}} \boldsymbol{e}_{i}^{T} \boldsymbol{e}_{i}+\frac{1}{k_{x} \sigma_{i}^{2}} \boldsymbol{\varepsilon}_{i}^{T} \boldsymbol{\varepsilon}_{i}\right]
$$


subject to (1). It is an constrained minimization problem and can be converted to a free minimization problem through introducing the Lagrange multipliers, i.e.

$$
\Phi=\sum_{i=1}^{N}\left[\frac{1}{k_{y} \sigma_{i}^{2}} \boldsymbol{e}_{i}^{T} \boldsymbol{e}_{i}+\frac{1}{k_{x} \sigma_{i}^{2}} \boldsymbol{\varepsilon}_{i}^{T} \boldsymbol{\varepsilon}_{i}+\lambda_{i}^{T}\left(\boldsymbol{y}_{i}-\boldsymbol{e}_{i}-\boldsymbol{t}-s \boldsymbol{R} \boldsymbol{x}_{i}+s \boldsymbol{R} \boldsymbol{\varepsilon}_{i}\right)\right]
$$

At the stationary points, which are candidates of minimizers, we have

$$
\begin{aligned}
& \frac{\partial \Phi}{\partial \boldsymbol{e}_{i}}=\frac{2}{k_{y} \sigma_{i}^{2}} \boldsymbol{e}_{i}-2 \lambda_{i}=0 \\
& \frac{\partial \Phi}{\partial \boldsymbol{\varepsilon}_{i}}=\frac{2}{k_{x} \sigma_{i}^{2}} \boldsymbol{\varepsilon}_{i}+2 s \boldsymbol{R}^{T} \lambda_{i}=0
\end{aligned}
$$

So we have

$$
\begin{aligned}
\boldsymbol{e}_{i} & =k_{y} \sigma_{i}^{2} \boldsymbol{\lambda}_{i} \\
\boldsymbol{\varepsilon}_{i} & =-s k_{x} \sigma_{i}^{2} \boldsymbol{R}^{T} \boldsymbol{\lambda}_{i}
\end{aligned}
$$

This stationary point is indeed minimizer because the Hessian matrices

$$
\begin{aligned}
\frac{\partial^{2} \Phi}{\partial \boldsymbol{e}_{i} \partial \boldsymbol{e}_{i}^{T}} & =\frac{2}{k_{y} \sigma_{i}^{2}} \boldsymbol{I} \\
\frac{\partial^{2} \Phi}{\partial \boldsymbol{\varepsilon}_{i} \partial \boldsymbol{\varepsilon}_{i}^{T}} & =\frac{2}{k_{x} \sigma_{i}^{2}} \boldsymbol{I}
\end{aligned}
$$

are both positive definite.

Substitute (8) into (1), with $\boldsymbol{R}^{T}=\boldsymbol{I}$ in mind, we have

$$
\boldsymbol{y}_{i}-\boldsymbol{t}-s \boldsymbol{R} \boldsymbol{x}_{i}-\left(k_{y}+k_{x} s^{2}\right) \sigma_{i}^{2} \lambda_{i}=0 \Leftrightarrow \lambda_{i}=\frac{1}{\left(k_{y}+k_{x} s^{2}\right) \sigma_{i}^{2}}\left(\boldsymbol{y}_{i}-\boldsymbol{t}-s \boldsymbol{R} \boldsymbol{x}_{i}\right)
$$

Substitute (10) into (8), then into (5), we have

$$
L=\frac{1}{k_{y}+k_{x} s^{2}} \sum_{i=1}^{N} \frac{1}{\sigma_{i}^{2}}\left(\boldsymbol{y}_{i}-\boldsymbol{t}-s \boldsymbol{R} \boldsymbol{x}_{i}\right)^{T}\left(\boldsymbol{y}_{i}-\boldsymbol{t}-s \boldsymbol{R} \boldsymbol{x}_{i}\right)
$$

The necessary condition of (11) with respect to $t$ is

$$
\frac{\partial L}{\partial \boldsymbol{t}}=\frac{1}{k_{y}+k_{x} s^{2}} \sum_{i=1}^{N} \frac{1}{\sigma_{i}^{2}}\left(-2 \boldsymbol{y}_{i}+2 s \boldsymbol{R} \boldsymbol{x}_{i}+2 \boldsymbol{t}\right)=0 \Leftrightarrow \boldsymbol{t}=\overline{\boldsymbol{y}}-s \boldsymbol{R} \boldsymbol{x}
$$

where the weighted means are

$$
\overline{\boldsymbol{y}}=\frac{\sum_{i=1}^{N} \frac{1}{\sigma_{i}^{2}} \boldsymbol{y}_{i}}{\sum_{i=1}^{N} \frac{1}{\sigma_{i}^{2}}}, \quad \boldsymbol{x}=\frac{\sum_{i=1}^{N} \frac{1}{\sigma_{i}^{2}} \boldsymbol{x}_{i}}{\sum_{i=1}^{N} \frac{1}{\sigma_{i}^{2}}}
$$

Note that (12) also suffice to be a minimizer because the Hessian matrix 


$$
\frac{\partial^{2} L}{\partial \boldsymbol{t} \partial \boldsymbol{t}^{T}}=\left(\frac{1}{k_{y}+k_{x} s^{2}} \sum_{i=1}^{N} \frac{1}{\sigma_{i}^{2}}\right) \boldsymbol{I}
$$

is positive definite.

Substitute (12) into (11), we have

$$
L=\frac{1}{k_{y}+k_{x} s^{2}} \sum_{i=1}^{N} \frac{1}{\sigma_{i}^{2}}\left(\Delta \boldsymbol{y}_{i}-s \boldsymbol{R} \Delta \boldsymbol{x}_{i}\right)^{T}\left(\Delta \boldsymbol{y}_{i}-s \boldsymbol{R} \Delta \boldsymbol{x}_{i}\right)=\frac{1}{k_{y}+k_{x} s^{2}}\left(a s^{2}-2 c s+b\right)
$$

where

$$
\begin{gathered}
\Delta \boldsymbol{y}_{i}=\boldsymbol{y}_{i}-\overline{\boldsymbol{y}}, \Delta \boldsymbol{x}_{i}=\boldsymbol{x}_{i}-\overline{\boldsymbol{x}} \\
a=\sum_{i=1}^{N} \frac{1}{\sigma_{i}^{2}}\left\|\Delta \boldsymbol{x}_{i}\right\|^{2}, b=\sum_{i=1}^{N} \frac{1}{\sigma_{i}^{2}}\left\|\Delta \boldsymbol{y}_{i}\right\|^{2} \\
c=\sum_{i=1}^{N} \frac{1}{\sigma_{i}^{2}} \Delta \boldsymbol{x}_{i}^{T} \boldsymbol{R}^{T} \Delta \boldsymbol{y}_{i}=\sum_{i=1}^{N} \frac{1}{\sigma_{i}^{2}} \Delta \boldsymbol{y}_{i}^{T} \boldsymbol{R} \Delta \boldsymbol{x}_{i}
\end{gathered}
$$

From (Chang 2015), with moderate $\boldsymbol{e}_{i}$ and $\boldsymbol{\varepsilon}_{i}, \boldsymbol{R} \Delta \boldsymbol{x}_{i}$ should be approximately parallel with $\Delta \boldsymbol{y}_{i}$, so $c>0$.

The necessary condition of minimizing (15) with respect to $s$ reads

$$
\frac{\partial L}{\partial s}=2 \frac{c k_{x} s^{2}+\left(a k_{y}-b k_{x}\right) s-c k_{y}}{\left(k_{y}+k_{x} s^{2}\right)^{2}}=0 \Leftrightarrow c k_{x} s^{2}+\left(a k_{y}-b k_{x}\right) s-c k_{y}=0
$$

So

$$
\begin{aligned}
& s_{1}=\frac{-d+f}{2 c k_{x}} \\
& s_{2}=\frac{-d-f}{2 c k_{x}}
\end{aligned}
$$

with

$$
\begin{aligned}
& d=a k_{y}-b k_{x} \\
& f=\sqrt{d^{2}+4 c^{2} k_{x} k_{y}}
\end{aligned}
$$

Note that $f>|d|>0$, so we can simply discard $s_{2}$ in (20) as in (Chang 2015) because it is negative. A further investigation about the Hessians evaluated at both solutions can tell that $s_{1}$ is a minimizer and $s_{2}$ is a maximizer, because

$$
\begin{aligned}
& \left.\frac{\partial^{2} L}{\partial s^{2}}\right|_{s=s_{1}}=\frac{f^{2}(f-d)}{2 c^{2} k_{x}\left(k_{y}+k_{x} s_{1}^{2}\right)^{3}}>0 \\
& \left.\frac{\partial^{2} L}{\partial s^{2}}\right|_{s=s_{2}}=\frac{-f^{2}(f+d)}{2 c^{2} k_{x}\left(k_{y}+k_{x} s_{2}^{2}\right)^{3}}<0
\end{aligned}
$$

We rearrange (15) as follows 


$$
L=\frac{1}{k_{y}+k_{x} s^{2}}\left(a s^{2}-2 c s+b\right)=a-G
$$

with

$$
G=\frac{d+2 c k_{x} s}{k_{y}+k_{x} s^{2}}
$$

Substitute $s_{1}$ into (24), we have

$$
G=\frac{d+2 c k_{x} s}{k_{y}+k_{x} s^{2}}=\frac{2 c^{2} k_{x} f}{(f-d) f} \stackrel{f \geq 0}{=} \frac{2 c^{2} k_{x}}{f-d} \stackrel{f}{=|d|} \frac{2 c^{2} k_{x}(f+d)}{f^{2}-d^{2}}=\frac{f+d}{2 k_{y}}
$$

From (23), minimizing the loss function $L$ is equivalent to maximizing the gain function $G$, and from (25), maximizing $G$ is equivalent to maximizing $f$, because $d$ and $k_{y}$ are independent with $R$. Further from the definition of $f$ in (21), maximizing $f$ is equivalent to maximizing $c$. It is routine to estimate $\boldsymbol{R}$ which maximizing $c$, and it can be found in several papers in the geodetic literature, e.g. those using the direction cosine matrix (Grafarend and Awange 2003; Teunissen 1984), and those using the unit quaternion (Sanso 1973; Shen et al. 2006). Note that same method have been proposed in other disciplines, e.g. crystallography (Mackenzie 1957), biometrics (Stephens 1979), computer vision (Arun et al. 1987; Horn 1987; Horn et al. 1988; Umeyama 1991), and aerospace (Markley 1988; Shuster and Oh 1981). For completeness, the algorithm of computing $\boldsymbol{R}$ as the direction cosine matrix will be summarized in the algorithmic table in the sequel.

To this moment, it would be of interest to consider two special cases, i.e. $k_{x} \rightarrow 0$ or $k_{y} \rightarrow 0$, which correspond to the Helmert transformation. For $k_{y} \rightarrow 0$, we have the following deduction, $d \rightarrow-b k_{x}, f \rightarrow b k_{x}, s_{1} \rightarrow b / c, G \rightarrow c^{2} / b$. As $c>0$, maximizing $c^{2} / b$ is equivalent to maximizing $c$. For $k_{x} \rightarrow 0$, the following deduction holds, $d \rightarrow a k_{y}, f \rightarrow a k_{y}, s_{1} \rightarrow c / a$, which is obtained to solve (19) directly, and from (15), $L \rightarrow\left(-c^{2}+a b\right) / a k_{y}$, so again, $\boldsymbol{R}$ should

\begin{tabular}{|c|c|}
\hline Step & Helmert model $\left(k_{x}=0\right) \quad$ Helmert model $\left(k_{y}=0\right)$ \\
\hline Problem formulation & Given: $\boldsymbol{x}_{i}$ and $\boldsymbol{y}_{i}$, To be estimated: $s, \boldsymbol{t}$, and $\boldsymbol{R}$. \\
\hline Data preprocessing & $\begin{aligned} \overline{\boldsymbol{y}} & =\frac{\sum_{i=1}^{N} \frac{1}{\sigma_{i}^{2}} \boldsymbol{y}_{i}}{\sum_{i=1}^{N} \frac{1}{\sigma_{i}^{2}}}, \overline{\boldsymbol{x}}=\frac{\sum_{i=1}^{N} \frac{1}{\sigma_{i}^{2}} \boldsymbol{x}_{i}}{\sum_{i=1}^{N} \frac{1}{\sigma_{i}^{2}}}, \Delta \boldsymbol{y}_{i}=\boldsymbol{y}_{i}-\overline{\boldsymbol{y}}, \Delta \boldsymbol{x}_{i}=\boldsymbol{x}_{i}-\overline{\boldsymbol{x}}, a=\sum_{i=1}^{N} \frac{1}{\sigma_{i}^{2}}\left\|\Delta \boldsymbol{x}_{i}\right\|^{2}, \\
b & =\sum_{i=1}^{N} \frac{1}{\sigma_{i}^{2}}\left\|\Delta \boldsymbol{y}_{i}\right\|^{2}, \boldsymbol{H}=\sum_{i=1}^{N} \frac{1}{\sigma_{i}^{2}} \Delta \boldsymbol{x}_{i} \Delta \boldsymbol{y}_{i}^{T}\end{aligned}$ \\
\hline Rotation estimation & $\boldsymbol{H}=\boldsymbol{U}\left[\begin{array}{ccc}\sigma_{1} & 0 & 0 \\
0 & \sigma_{2} & 0 \\
0 & 0 & \sigma_{3}\end{array}\right] \boldsymbol{V}^{T}, \boldsymbol{R}=\boldsymbol{V}\left[\begin{array}{ccc}1 & 0 & 0 \\
0 & 1 & 0 \\
0 & 0 & \operatorname{det}[\boldsymbol{U}] \operatorname{det}[\boldsymbol{V}]\end{array}\right] \boldsymbol{U}^{T}$ \\
\hline Scale estimation & $\begin{array}{l}c=\operatorname{trace}[\boldsymbol{R} \boldsymbol{H}] \\
d=a k_{y}-b k_{x} \\
f=\sqrt{d^{2}+4 c^{2} k_{x} k_{y}} \\
s=\frac{-d+f}{2 c k_{x}}\end{array}$ \\
\hline Translation estimation & $t=\overline{\boldsymbol{y}}-s \boldsymbol{R} \boldsymbol{x}$ \\
\hline
\end{tabular}
maximize $c$.

Table 1 Algorithms for Helmert and symmetric Helmert datum transformations 
To summarize, for both Helmert and symmetric Helmert models, the rotation parameters in the LS solutions are the same to each other, because they are both to maximize $c$ which is independent of $k_{x}$ and $k_{y}$.

To facilitate implementation, the algorithm is summarized step by step in Table 1. Note that in the "Translation estimation" step, it does not necessarily imply that the translational parameters are the same for the three models, because thought some arguments, e.g. the rotational parameter, are the same for the three models, other argument, e.g. the scale parameters are different for these models.

Note that in the table, the equation $\boldsymbol{H}=\boldsymbol{U}\left[\begin{array}{ccc}\sigma_{1} & 0 & 0 \\ 0 & \sigma_{2} & 0 \\ 0 & 0 & \sigma_{3}\end{array}\right] \boldsymbol{V}^{T}$ means the singular value decomposition of $\boldsymbol{H}$, i.e., the columns of the orthogonal matrices $\boldsymbol{U}$ and $\boldsymbol{V}$ are the left and right singular vectors of $\boldsymbol{H}$ respectively, and the $\sigma_{1} \geq \sigma_{2} \geq \sigma_{3} \geq 0$ are the singular values of $\boldsymbol{H}, \operatorname{det}[]$ and trance[] denote the determinant and the trace of a matrix respectively.

\section{Concluding remarks}

In this note, the $3 \mathrm{D}$ similarity datum transformation problem is studied. The LS solution to the Helmert and symmetric Helmert models are derived in a unified framework. The rotational invariant covariance structure is adopted. The main findings are that the closedform solutions are derived, and that in the solutions to both models, the rotational parameters are the same. It can be considered as the extension of 3D symmetric Helmert transformation with naïve identity covariance studied in a previous work (Chang 2015), and a counterpart of the 2D symmetric Helmert transformation with rotational invariant covariance studied in (Teunissen 1988).

Acknowledgments This work was supported in part by National Natural Science Foundation of China (No. 41404001).

\section{References}

Arun KS, Huang TS, Blostein SD (1987) Least-squares fitting of two 3-D point sets. IEEE Trans Pattern Anal Mach Intell 5:698-700

Chang G (2015) On least-squares solution to 3D similarity transformation problem under Gauss-Helmert model. J Geodesy 89(6):573-576

Fang X (2014) A total least squares solution for geodetic datum transformations. Acta Geod et Geophys 49(2):189-207

Felus YA, Burtch RC (2009) On symmetrical three-dimensional datum conversion. GPS Solut 13(1):65-74

Grafarend EW, Awange JL (2003) Nonlinear analysis of the three-dimensional datum transformation [conformal group C7(3)]. J Geodesy 77:66-76

Horn BK (1987) Closed-form solution of absolute orientation using unit quaternions. J Opt Soc Am A 4(4):629-642

Horn BK, Hilden HM, Negahdaripour S (1988) Closed-form solution of absolute orientation using orthonormal matrices. J Opt Soc Am A 5(7):1127-1135

Kanatani K, Niitsuma H. (2012a). Optimal Computation of 3-D Similarity from Space Data with Inhomogeneous Noise Distributions. Memoirs of the Faculty of Engineering, Okayama University, 46 (1-9)

Kanatani K, Niitsuma H (2012b) Optimal computation of 3-D similarity: Gauss-Newton vs. Gauss-Helmert. Comput Stat Data Anal 56(12):4470-4483 
Li B, Shen Y, Lou L (2013) Noniterative datum transformation revisited with two-dimensional affine model as a case study. J Surv Eng 139(4):166-175

Mackenzie J (1957) The estimation of an orientation relationship. Acta Crystallogr A 10(1):61-62

Markley FL (1988) Attitude determination using vector observations and the singular value decomposition. J Astronaut Sci 36(3):245-258

Neitzel F (2010) Generalization of total least-squares on example of unweighted and weighted 2D similarity transformation. J Geodesy 84(12):751-762

Sanso F (1973) An exact solution of the roto-translation problem. Photogrammetria 29:203-216

Schaffrin B (2006) A note on constrained total least-squares estimation. Linear Algebra Appl 417(1):245-258

Schaffrin B, Felus YA (2008) On the multivariate total least-squares approach to empirical coordinate transformations. Three algorithms. J Geodesy 82(6):373-383

Schaffrin B, Wieser A (2008) On weighted total least-squares adjustment for linear regression. J Geodesy 82(7):415-421

Shen Y, Chen Y, Zheng D-H (2006) A quaternion-based geodetic datum transformation algorithm. J Geodesy 80(5):233-239

Shuster MD, Oh S (1981) Three-axis attitude determination from vector observations. J Guid Control Dyn 4(1):70-77

Stephens M (1979) Vector correlation. Biometrika 66(1):41-48

Teunissen PJG (1984). Generalized inverses, adjustment, the datum problem and S-transformations. In: International School of Geodesy, 3rd Course: Optimization and Design of Geodetic Networks, EriceTrapani-Sicily, pp 1-49

Teunissen PJG (1985) The geometry of geodetic inverse linear mapping and non-linear adjustment. Netherlands Geodetic Commision. Publications on Geodesy, New Series, pp 1-186

Teunissen PJG (1986) Adjusting and testing with the models of the affine and similarity transformation. Manuscripta Geodaet 11:214-225

Teunissen PJG (1988) The non-linear 2D symmetric Helmert transformation: an exact non-linear leastsquares solution. Bulletin Géodésique 62(1):1-16

Teunissen PJG. (1989). A note on the bias in the symmetric Helmert transformation. Festschrift Torben Krarup: 335-342

Umeyama S (1991) Least-squares estimation of transformation parameters between two point patterns. IEEE Trans Pattern Anal Mach Intell 13(4):376-380

Xu P, Liu J, Shi C (2012) Total least squares adjustment in partial errors-in-variables models: algorithm and statistical analysis. J Geodesy 86(8):661-675

Yang Y (1999) Robust estimation of geodetic datum transformation. J Geodesy 73(5):268-274 